\title{
Uso de estratégias cognitivas diante de queixas subjetivas de memória em idosos saudáveis
}

\section{Use of cognitive strategies on subjective memory complaints in healthy seniors}

\author{
Rafael Gomes Firmino ${ }^{1}$, Isabela Lemos Arteiro Ribeiro Lins ${ }^{2}$, \\ Bernardino Fernández-Calvo ${ }^{3}$
}

http://dx.doi.org/10.11606/issn.2238-6149.v30i2p102-109

Firmino RG, Arteiro ILR, Fernández-Calvo BF. Uso de estratégias cognitivas diante de queixas subjetivas de memória em idosos saudáveis. Rev Ter Ocup Univ São Paulo. 2020 maioago.;30(2):102-9.

RESUMO: Queixas Subjetivas de Memória (QSM) tem sido alvo de pesquisas, sendo apontadas como corriqueiras no cotidiano de pessoas idosas. O presente estudo teve por objetivo verificar se há correlação entre as Queixas Subjetivas de Memória evidenciadas pelo Questionário de Memória Prospectiva e Retrospectiva (QMPR) e as variáveis: Escolaridade (E), Reserva Cognitiva (RC), Funcionalidade (FAQ) e o uso de Estratégias (MMQ). Trata-se de um estudo correlacional, de abordagem quantitativa com delineamento "ex post facto". A amostra foi composta por 64 idosos, tendo idade média de 69 anos $(\mathrm{DP}=6,45)$, sendo estes recrutados em espaços de convivência situados nas cidades de João Pessoa e Guarabira, por meio da divulgação de folders e via redes sociais. As coletas foram realizadas nos seguintes locais: Centro de Convivência da Pessoa Idosa (CCI), no bairro Castelo Branco-JP; Centro de Vivência da Universidade Federal da Paraíba (UFPB); Grupo de hidroginástica para terceira idade,

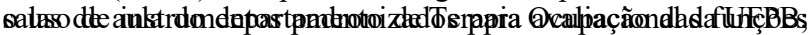

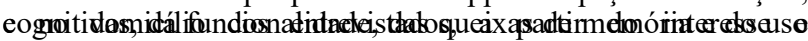
defiestidadégiade aléshockampentol. słpeliiedensegráfico. Os resultados indicaram correlação positiva entre as variáveis: Escolaridade (E), Reserva cognitiva (RC), Funcionalidade (FAQ) e Queixas de Memória Prospectiva e Retrospectiva (QMPR) com o uso de estratégias de memória (MMQ). Contudo, faz-se necessário destacar a presença evidente de QSM nos idosos, bem como a significativa correlação entre estas e o uso de estratégias cognitivas, sendo as queixas de memória prospectivas mais predominantes que as queixas retrospectivas. Concluímos que idosos que apresentam maior uso de estratégias na vida diária tendem a melhor perceber os relatos de suas queixas. Sugere-se que maiores pesquisas sobre a temática invistam em intervenções para o treino do uso de estratégias de memória em idosos cognitivamente saudáveis que relatem QSM na vida diária, para um melhor bem-estar e qualidade de vida.

DESCRITORES: Transtornos da memória; Reserva cognitiva; Inquéritos e questionários; Idoso.
Firmino RG, Arteiro ILR, Fernández-Calvo BF. Use of cognitive strategies on subjective memory complaints in healthy seniors. Rev Ter Ocup Univ São Paulo. 2020 May-Aug.;30(2):102-9.

ABSTRACT: Memory Subjective Complaints (MSC) has been the subject of researches, being pointed it out as unexceptional in the daily life of elderly people. The objective of this study was to verify if there is a correlation between Memory Subjective Complaints evidenced by the Prospective and Retrospective Memory Questionnaire (PRMQ) and the variables: Schooling (S), Cognitive Reserve (CR), Functionality (FAQ) and the use of Strategies (MMQ). This is a correlational study, with a quantitative approach with an "ex post facto" lineation. The sample consisted of 64 elderly people, with a mean age of 69 years $(\mathrm{SD}=6.45)$, who were recruited in a social space located in the cities of João Pessoa and Guarabira, through the dissemination of folders and via social networks. The collections carried out in the following places: Centro de Convivência da Pessoa Idosa (CCI) in the district Castelo Branco-JP, Centro de Vivência da Universidade Federal da Paraíba (UFPB), Group of hydrogymnastics for the elderly, classrooms of the Department of Therapy Occupational of the UFPB and in the domicile of the interviewed ones based in the interest and difficulties of displacement. The use of standardized instruments were applied for the evaluation of cognitive functions, of functionality, memory complaints and the use of strategies, and the sociodemographic profile as well. The results indicated a positive correlation between the variables: Schooling (E), Cognitive Reserve (CR), Functionality (FAQ), and Prospective and Retrospective Memory Complaints (PRMC) using memory strategies (MMQ). However, it is necessary to highlight the evident presence of MSC in the elderly, the significant correlation between these and the use of cognitive strategies as well, being the prospective memory complaints more prevalent than retrospective complaints. We conclude that elderly people who present greater use of strategies in daily life tend to perceive the reports of their complaints better. It is suggested more researches on the theme, investing in interventions for the training of the use of memory strategies in cognitively healthy elders who report MSC in daily life, for better well-being and quality of life.

KEYWORDS: Memory disorders; Cognitive reserve; Surveys and questionnaires; Aged.

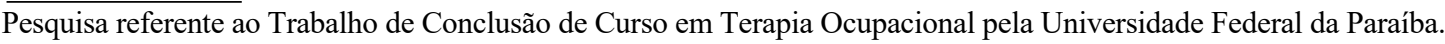

1. Graduando em Terapia Ocupacional pela Universidade Federal da Paraíba. https://orcid.org/0000-0002-6144-8445. Email: rafaelgomesufpb@gmail.com ou rafaelgomesto.ufpb@gmail.com.

2. Professora Adjunta na Universidade Federal da Paraíba. Doutora em Psicologia Clínica pela Universidade Católica do Pernambuco. https://orcid.org/0000-0002-3748-8463. E-mail: isabelalemos@gmail.com.

3. Professor Adjunto I na Universidade Federal da Paraíba. Doutor em Psicologia Clínica e da Saúde pela Universidade de Salamanca. https://orcid.org/0000-0001-8080-5578. E-mail: bfcalvo@usual.es.

Endereço para correspondência: Rafael Gomes Firmino. Rua. Antonieta M. Mendes, n.124. Santa Terezinha - Guarabira/PB. 


\section{INTRODUÇÃO}

A tualmente, tem-se observado um aumento crescente do envelhecimento populacional, fator este atribuído à redução dos índices de natalidade e mortalidade, ocasionando uma maior expectativa de vida no Brasil e no mundo. Segundo dados mais recentes levantados em pesquisa do Instituto Brasileiro de Geografia e Estatística, a população brasileira ganhou 4,8 milhões de idosos desde o ano de 2012, chegando a um quantitativo de 30,2 milhões, em $2017^{1}$.

Projeções futuras apontam para uma sociedade cada vez mais idosa, e em consequência precisa-se estar atento ao surgimento de doenças e/ou patologias relacionadas ao envelhecimento como um todo, sendo importante também salientar a emergência de atenção para o envelhecimento cognitivo, em especial aos conteúdos da memória. O presente estudo pauta-se na necessidade atual de se conhecer mais sobre as Queixas Subjetivas de Memória (QSM), o uso de estratégias cognitivas e a correlação destas com outras variáveis em idosos cognitivamente saudáveis.

As QSM são conhecidas como auto relatos das pessoas de qualquer faixa etária, realidade socioeconômica e nível intelectual ${ }^{2}$ sobre o funcionamento da memória, sendo mais comuns no cotidiano de pessoas idosas ${ }^{3}$, e podem estar relacionadas a queixas de memória prospectivas e retrospectivas, sendo a primeira voltada para a dificuldade em recordar atividades que deveriam ser realizadas no futuro próximo e, a segunda, para limitações em recordar atividades feitas no passado.

Balash et al. ${ }^{4}$ enfatizam que as queixas subjetivas são mais corriqueiras no cotidiano de pessoas idosas. A relação entre QSM e reserva cognitiva ( $\mathrm{RC}$ ) tem sido escassamente estudada na literatura, entretanto, nem todos os tipos de QSM foram avaliados nessas pesquisas, abrindo um campo de estudo comparativo entre QSM e RC.

Perquin et al. ${ }^{5}$ apontam que a ausência de QSM em população idosa pode estar associada a fatores protetivos, tais como o grau de escolaridade e o envolvimento em atividades intelectuais, por exemplo.

Além das evidências de que as QSM afetam a qualidade de vida dos idosos, autores também demonstram que estas podem servir como preditores de prejuízos cognitivos e processos demenciais ${ }^{6}$. Hipóteses apontam que influências negativas na meta-memória de pessoas idosas (descrenças quanto à capacidade de memorizar) repercutem diretamente no uso de estratégias cognitivas ${ }^{7}$. Outras sugerem que idosos apresentam pouca eficiência no seu estilo cognitivo por usarem de modo reduzido tais estratégias ${ }^{8}$.
O debate de temáticas como esta também suscita a urgência de se buscar cada dia mais uma assistência integrada e multiprofissional, e o terapeuta ocupacional é um dos profissionais aptos a atuar com o público em foco.

A Terapia Ocupacional (TO) é caracterizada como profissão da saúde, e tem por objetivo a emancipação, autonomia e independência das pessoas em suas Atividades de Vida Diária (AVD), tem relevante papel na reabilitação das funções físicas, mentais e cognitivas. Atua na prevenção de futuros agravos e na promoção da qualidade de vida do público idoso, auxiliando na manutenção, recuperação e/ ou restauração da funcionalidade para assim possibilitar e favorecer o engajamento ocupacional ${ }^{9}$.

A motivação para a presente pesquisa surgiu a partir do interesse pela temática do envelhecimento e de experiências em disciplinas teórico-práticas do curso e de outras vivências acadêmicas. $\mathrm{O}$ contato com o público idoso, mais precisamente no ambulatório de Geriatria do Hospital Universitário Lauro Wanderley (HULW), onde desempenhei o papel de estagiário, incentivou-me a buscar compreender mais sobre os conteúdos da memória e do envelhecimento cognitivo.

Levando em consideração o aumento das QSM na população idosa faz-se necessário debruçar-se mais sobre estas, a fim de conhecê-las e verificar as repercussões na vida cotidiana das pessoas, além de aprofundar sobre os diversos aspectos que estão relacionados às mesmas. Portanto, a presente pesquisa levanta o seguinte questionamento: Quais são as variáveis de estudo que se correlacionam com as QSM em idosos considerados cognitivamente saudáveis? O uso de estratégias cognitivas tem correlação significativa com as queixas subjetivas de memória?

Acredita-se que as QSM estão significativamente correlacionadas com fatores como: Escolaridade (envolvimento em atividades educacionais), Reserva Cognitiva, Funcionalidade, e uso de Estratégias de Memória (medidas compensatórias auxiliares internas ou externas). Queixas de memória prospectiva surgirão com maior destaque se comparadas às retrospectivas.

Considerando a relevância dos estudos sobre o uso de estratégias de memória adotadas por idosos saudáveis que apresentam QSM, a pesquisa justifica-se pela necessidade de se ampliar a compreensão atual sobre essas estratégias e como a correlação com outras variáveis podem modular as queixas de memória, visto que o auto relato do paciente possui relevância nas decisões clínicas no contexto do envelhecimento. Assim, os resultados do presente estudo poderão contribuir na reformulação de conceitos de prevenção, diagnóstico, tratamento de alterações cognitivas 
em idosos saudáveis, além da promoção de saúde e avanços na ciência.

Portanto, o estudo teve por objetivo verificar se há correlação entre as QSM demonstradas pelo Questionário de Memória Prospectiva e Retrospectiva (QMPR) e as seguintes variáveis: Escolaridade (E), Reserva Cognitiva (RC), Funcionalidade (FAQ) e uso de Estratégias (MMQ). Para tal investigação foi necessário avaliar cognitivamente os sujeitos da pesquisa a fim de selecionar aqueles que relataram queixas de memória, mas que não apresentaram transtornos neurocognitivos.

\section{PROCEDIMENTOS METODOLÓGICOS}

O presente estudo caracteriza-se por seu delineamento "ex post facto" de tipo correlacional de abordagem quantitativa. Para fins do alcance de seus objetivos foram analisadas as seguintes variáveis: Escolaridade (E), Reserva Cognitiva (RC), Funcionalidade (FAQ), Memória Prospectiva e Retrospectiva (QMPR) e o uso de estratégias de memória (MMQ).

Os idosos participantes da pesquisa foram recrutados em espaços de convivência situados nas cidades de João Pessoa e Guarabira (região do agreste paraibano), por meio da divulgação de folders e via redes sociais. As coletas foram realizadas nos seguintes locais: Centro de Convivência da Pessoa Idosa (CCI), no bairro Castelo Branco-JP; Centro de Vivência da UFPB; Grupo de hidroginástica para terceira idade, salas de aula do departamento de Terapia Ocupacional da Universidade Federal da Paraíba (UFPB); e no domicílio dos entrevistados, considerando o interesse e as dificuldades de deslocamento.

O estudo realizou-se de agosto do ano de 2018 a abril de 2019.

\section{Amostra}

A amostra da pesquisa contou com a participação de pessoas idosas de ambos os sexos que preenchessem os seguintes critérios de elegibilidade:

\section{Critérios de Inclusão}

Ter idade igual ou superior a 60 anos; não possuir comprometimento cognitivo moderado ou grave, evidenciado pela pontuação igual ou inferior a 23, obtida no Mini Exame do Estado Mental (MEEM); ou por um escore maior ou igual a 4, na escala FAQ de Atividades de Vida Diária (AVD) de Pfeffer et al. ${ }^{13}$; e não institucionalizados.

\section{Critérios de Exclusão}

Possuir diagnóstico de transtorno neurocognitivo maior de acordo com os critérios estabelecidos pelo DSM-V ou enfermidades psiquiátricas graves; apresentar dificuldades de comunicação ou compreensão (afasias); possuir deficiências sensoriais que interferissem no momento da entrevista.

Com base nesses critérios, de 90 idosos entrevistados, foram excluídos 26 da pesquisa por não responderem aos princípios de inclusão, ou seja, por não apresentarem escore maior que 23, no MEEM, considerando-se também a nota de corte 20 para os não escolarizados ${ }^{10}$, e aqueles que obtiveram pontuação igual ou maior a 4 na escala FAQ de Atividades de Vida Diária - Pfeffer et al. ${ }^{13}$. A amostra final do estudo correspondeu a um quantitativo de 64 pessoas idosas.

\section{Instrumentos e procedimentos}

O procedimento utilizado nesse estudo constou de uma fase avaliativa, em que ocorreu a aplicação dos questionários com cada participante de modo individualizado. As avaliações tiveram durabilidade média de 30 minutos.

Foram aplicados os seguintes instrumentos: (1) Questionário sociodemográfico com dados de sexo, idade, escolaridade, profissão, entre outros; (2) Questionário de saúde adaptado com entrevista clínica para informações objetivas (hipertensão arterial, diabetes, hipercolesterolemia, por exemplo) e subjetivas (percepção da saúde, memória e qualidade de vida); (3) Questionário de Memória Prospectiva e Retrospectiva - QMPR ${ }^{11}$, composto por 16 itens, tendo oito categorias, envolvendo diferentes aspectos da memória prospectiva (curto prazo) e retrospectiva (curto e longo prazo); (4) Mini Exame do Estado Mental (MEEM) ${ }^{12}$, utilizado para a avaliação das funções cognitivas, apresentando 18 itens e pontuação máxima igual a 30; (5) FAQ - Escala de Atividades de Vida Diária ${ }^{13}$, para avaliação da funcionalidade e aspectos como: autocuidado, alimentação, higiene, vestuário e mobilidade, permitindo também uma pontuação máxima de 30 pontos; (6) Escala Hospitalar de Ansiedade e Depressão $0^{14}$ para controle da amostra, composta por 14 itens aleatórios sobre ansiedade e depressão, com a consideração dos sintomas apresentados pelos entrevistados nos últimos 30 dias; (7) Questionário de Reserva Cognitiva - QRC ${ }^{15}$, constituído de três fatores: atividades intelectuais, físicas e sociais; (8) Questionário de Estratégias de Memória (MMQ - Estratégia), contemplando 19 itens, considerando-se as duas últimas semanas e a frequência de uso, quanto maior a pontuação melhor a utilização de estratégias mnemônicas ${ }^{16}$. 
Optou-se pelo uso de tais instrumentos para uma seleção minuciosa e detalhada dos participantes com a finalidade de alcançar o objetivo do estudo, considerando também a relevância de uma avaliação ampla e integral para os sujeitos.

\section{Análise estatística dos dados}

Os dados coletados foram submetidos e analisados através do programa estatístico SPSS, versão 22.0. Realizaramse as seguintes análises: (1) Estatística Descritiva: média (M) e Desvio-Padrão (DP) das características sociodemográficas (Idade, Sexo, Escolaridade, Atividade Laboral e Renda) e clínicas; (2) Correlação de Pearson entre o escore obtido no QMPR total e específicos (QMPR-P e QMPR-R), e as demais variáveis: Escolaridade (E), Reserva Cognitiva (RC), Funcionalidade (FAQ) e uso de Estratégia (MMQ); (3) Test t para análise comparativa entre os grupos que apresentaram uso elevado e reduzido de estratégias. Em todos os testes estatísticos, o nível de significância admitido foi $\mathrm{p}<0,05$.

\section{Aspectos éticos}

O presente estudo foi avaliado e aprovado pelo Comitê de Ética em Pesquisa envolvendo seres humanos do Centro de Ciências da Saúde/CCS-UFPB, de acordo com o que normatiza a Resolução 196/96 do Conselho Nacional de Saúde, com número de processo CAAE: 03419212.4.0000.5188. Todos os participantes assinaram um Termo de Consentimento Livre e Esclarecido (TCLE), sendo assegurados da não identificação e do sigilo das informações. Os aspectos éticos foram respeitados de acordo com o que preconiza a Resolução 466/2012, do Conselho Nacional de Saúde (CNS, 2013).

\section{RESULTADOS}

A Tabela 1 apresenta o perfil sociodemográfico dos participantes da pesquisa com ênfase em aspectos como: sexo, escolaridade, atividade laboral e renda. Dos 64 entrevistados incluídos na amostra, 51 (79,7\%) são do sexo feminino, e 13 (20,3\%), do sexo masculino. Em relação ao nível de escolaridade, 13 (20,3\%) possuem ensino superior, $15(23,4 \%)$ não são escolarizados, $6(9,4 \%)$ desenvolveram leitura e escrita, $14(21,9 \%)$ estudaram até o quinto ano incompleto, $7(10,9 \%)$ concluíram o ensino fundamental e $9(14,1)$ finalizaram o ensino médio. Desses idosos 52 $(81,3 \%)$ são aposentados e $42(65,5 \%)$ possuem uma renda de até dois salários mínimos.

Tabela 1 - Descrição do perfil sociodemográfico

\begin{tabular}{lcc}
\hline & N & \% \\
\hline Sexo & 51 & 79,7 \\
Feminino & 13 & 20,3 \\
Masculino & & \\
Escolaridade & 15 & 23,4 \\
Não estudou & 6 & 9,4 \\
Ler e escrever & 14 & 21,9 \\
Até o 5 ano incompleto & & \\
Continua & 7 & 10,9 \\
Ensino fundamental completo & 9 & 14,1 \\
Ensino médio completo & 13 & 20,3 \\
Ensino superior & & \\
Atividade Laboral & 52 & 81,3 \\
Aposentado & 9 & 14,1 \\
Ativo & 3 & 4,7 \\
Não se aplica & & \\
Renda & 42 & 65,6 \\
Até dois salários mínimos & 15 & 23,4 \\
De três a quatro salários mínimos & 4 & 6,3 \\
De cinco a seis salários mínimos & 2 & 3,1 \\
De sete a oito salários mínimos & 1 & 1,6 \\
De nove a dez salários mínimos & 64 & 100 \\
Total & & \\
\hline
\end{tabular}

Fonte: Elaboração própria.

Na Tabela 2 são apresentadas as médias e desviospadrão das variáveis de estudo. Observou-se que a média de idade amostral foi de 69,38 (DP=6,45). Com base no Questionário de Reserva Cognitiva (QRC) a média para atividade intelectual foi de $8,48(\mathrm{DP}=4,22)$, para atividade física 1,50 $(\mathrm{DP}=1,57)$ e para atividade social 4,48 $(\mathrm{DP}=1,99)$, obtendo, assim, um valor médio de 14,44 $(\mathrm{DP}=5,78) \mathrm{em}$ seu escore total. 
Firmino RG, et al. Uso de estratégias cognitivas. Rev Ter Ocup Univ São Paulo. 2020 maio/ago.;30(2):102-9.

Tabela 2 - Estatística Descritiva: Médias e desvios-padrão

\begin{tabular}{lcc}
\hline & M & DP \\
\hline Idade & 69,38 & 6,45 \\
QRC-Atividade Intelectual & 8,48 & 4,22 \\
QRC-Atividade Física & 1,50 & 1,57 \\
QRC-Atividade Social & 4,48 & 1,99 \\
QRC-TOTAL & 14,44 & 5,78 \\
QMPR-P & 13,34 & 4,78 \\
QMPR-R & 11,14 & 3,76 \\
QMPR-T & 24,48 & 7,88 \\
HAD-A & 3,09 & 3,42 \\
HAD-D & 1,54 & 2,59 \\
HAD-TOTAL & 4,64 & 5,47 \\
MEEM & 25,38 & 2,79 \\
FAQ & 0,23 & 0,72 \\
MMQ Estratégia & 14,02 & 9.79 \\
\hline
\end{tabular}

Fonte: Elaboração própria. Legenda: $\mathrm{M}=$ Média; $\mathrm{DP}=$ Desvios-padrão; $\mathrm{QRC}=$ Questionário de Reserva Cognitiva; QMPR-P $=$ Questionário de memória prospectiva; $\mathrm{QMPR}-\mathrm{R}=$ Questionário de memória retrospectiva; QMPR-T = Questionário total de memória prospectiva e retrospectiva; HAD = Escala Hospitalar de Ansiedade e Depressão; MEEM = Mini Exame do Estado Mental; FAQ = Escala de Atividade de Vida Diária; $\mathrm{MMQ}=$ Questionário Multifatorial de Memória.

Em relação à sintomatologia ansiosa e depressiva, mensurada através da Escala Hospitalar de Ansiedade e Depressão (HAD) percebeu-se que não há a presença de ansiedade e depressão na amostra, devido terem obtido pontuação abaixo da nota de corte (maior ou igual a 9).

No Mini Exame do Estado Mental (MEEM) a média obtida pela amostra foi de 25,38 (DP=2,79) e na Escala FAQ de Atividades de Vida Diária obteve-se um resultado médio de $0,23(\mathrm{DP}=0,72)$ para a funcionalidade. $\mathrm{O}$ uso de estratégias cognitivas mensurado a partir do Questionário Multifatorial de Memória - MMQ teve uma média de $14,02(\mathrm{DP}=9,79)$.

No Questionário de Memória Prospectiva e Retrospectiva (QMPR) os idosos apresentaram significativamente maiores queixas cognitivas relacionadas à memória prospectiva $(\mathrm{M}=13,34, \mathrm{DP}=4,78)$. As queixas de memória retrospectiva foram demonstradas por um valor menor $(\mathrm{M}=11,14, \mathrm{DP}=3,76)$. Sendo assim, queixas de memória prospectiva estão mais relacionadas com o cotidiano de pessoas idosas $[(63)=5,080) \mathrm{P}<0,001]$.

A Tabela 3 apresenta a correlação de Pearson entre as variáveis de estudo e seus respectivos valores. Com base nos resultados observou-se que as queixas de memória e as variáveis Escolaridade (E), Funcionalidade (FAQ) e Reserva Cognitiva (RC) correlacionaram-se positivamente com o uso de estratégias cognitivas.

$\mathrm{Na}$ Tabela 4 apresenta-se os resultados obtidos pelo Teste $t$, em que se realizou um comparativo entre os grupos que apresentaram uso reduzido e elevado de estratégias. Percebeu-se que aqueles que utilizaram maiores estratégias cognitivas relataram mais queixas de memória, sejam elas prospectivas ou retrospectivas.

Tabela 3 - Correlação de Pearson entre as variáveis de estudo

\begin{tabular}{|c|c|c|c|c|c|c|c|c|}
\hline & E. & QRC & FAQ & MMSE & QMPR-P & QMPR- R & QMPR-T & MMQ \\
\hline E. & 1 & $0,84 * *$ & NS & $0,69 * *$ & NS & NS & NS & $0,40 * *$ \\
\hline QRC & & 1 & NS & $0,55 * *$ & NS & NS & NS & $0,31^{*}$ \\
\hline FAQ & & & 1 & NS & $0,28 *$ & $0,49 * *$ & $0,40 * *$ & $0,27^{*}$ \\
\hline MEEM & & & & 1 & NS. & NS. & NS. & $0,39 * *$ \\
\hline QMPR-P & & & & & 1 & $0,69 * *$ & $0,93 * *$ & $0,49 * *$ \\
\hline QMPR-R & & & & & & 1 & $0,93 * *$ & $0,55 * *$ \\
\hline QMPR-T & & & & & & & 1 & $0,56 * *$ \\
\hline MMQ & & & & & & & & 1 \\
\hline
\end{tabular}

Fonte: Elaboração própria. *p $<0,05 ; * * \mathrm{p}<0,01$. Legenda: $\mathrm{E}=$ Escolaridade; $\mathrm{QRC}=$ Total Questionário de Reserva Cognitiva; $\mathrm{FAQ}=$ Escala de Atividade de Vida Diária; MEEM = Mini Exame do Estado Mental; QMPR-P = Questionário de memória prospectiva; QMPR-R = Questionário de memória retrospectiva; QMPR-T = Questionário total de memória prospectiva e retrospectiva; MMQ = Estratégia: Questionário Multifatorial de Memória; NS = Não teve significância. 
Firmino RG, et al. Uso de estratégias cognitivas. Rev Ter Ocup Univ São Paulo. 2020 maio/ago.;30(2):102-9.

Tabela 4 - Test t. Comparativo entre os grupos com altas e baixas estratégias

\begin{tabular}{|c|c|c|c|c|c|c|}
\hline & $\begin{array}{c}\text { USO DE } \\
\text { ESTRATÉGIAS }\end{array}$ & $\mathbf{N}$ & M & DP & $\mathbf{T}$ & $\mathbf{P}$ \\
\hline \multirow[t]{2}{*}{ QMPR_P } & Baixa & 33 & 11,18 & 3,609 & $-4,191$ & 0,063 \\
\hline & Alta & 31 & 15,65 & 4,855 & & \\
\hline \multirow[t]{2}{*}{ QMPR_R } & Baixa & 33 & 9,61 & 2,193 & $-3,683$ & 0,001 \\
\hline & Alta & 31 & 12,77 & 4,395 & & \\
\hline \multirow[t]{2}{*}{ QMPR_T } & Baixa & 33 & 20,79 & 4,833 & $-4,399$ & 0,002 \\
\hline & Alta & 31 & 28,42 & 8,632 & & \\
\hline
\end{tabular}

Fonte: Elaboração própria. Legenda: $\mathrm{N}$ = número de sujeitos; $\mathrm{M}=$ Média; $\mathrm{DP}=$ Desvio Padrão; QMPR-P = Questionário de memória prospectiva; QMPR-R = Questionário de memória retrospectiva; QMPR-T = Questionário total de memória prospectiva e retrospectiva; $\mathrm{T}=$ Test; $\mathrm{P}=$ Significância.

\section{DISCUSSÃO}

De acordo com os achados do estudo, verificou-se a alta prevalência de QSM nos idosos da amostra, além de correlação significante entre o uso de estratégias de memória adotadas na vida diária e as variáveis: Escolaridade (E), Reserva Cognitiva (RC), Funcionalidade (FAQ) e Memória Prospectiva e Retrospectiva (QMPR).

Em relação aos dados sociodemográficos, conforme apresentado na tabela 1, percebeu-se uma predominância do sexo feminino, que atingiu 79,7\% da amostra, além de uma considerável presença de idosos não escolarizados, baixa renda e aposentados. Estes resultados vêm corroborar parcialmente com outros achados, em que o sexo feminino e a baixa escolaridade estão sendo associados a uma maior prevalência de Queixas Subjetivas de Memória (QSM) ${ }^{17}$.

Conforme o questionário de Reserva Cognitiva (RC) aplicado, os participantes da pesquisa relataram ter baixo envolvimento em atividades intelectuais, físicas e sociais, configurando-se em uma problemática, já que o reduzido engajamento em tais atividades pode repercutir negativamente na manutenção e melhoria da capacidade cognitiva no cotidiano dos sujeitos. Tal informação é corroborada por estudo desenvolvido por Perquin et al. ${ }^{5}$ que sugere que uma elevada RC reduz a prevalência de queixas de memória.

A Classificação Internacional de Funcionalidade, Incapacidade e Saúde - $\mathrm{CIF}^{18}$ define a funcionalidade como aquela que abarca todas as funções do corpo e as capacidades das pessoas em realizarem suas atividades diárias, de acordo com a interação dinâmica entre suas condições de saúde e os fatores contextuais (ambientais e pessoais). Um dado também relevante, evidenciado na Tabela 2 está relacionado à escala FAQ de Atividades de Vida Diária (AVD) em que se destaca a funcionalidade preservada da amostra, sendo esta fundamental para o desempenho em suas ocupações e na execução de atividades que demandam esforços físicos, mentais e cognitivos.

É importante enfatizar que a avaliação e realização das Atividades de Vida Diárias (AVD) servem como critério fundamental para a diferenciação e maior fidedignidade de diagnósticos em pessoas com Comprometimento Cognitivo Leve (CCL) e quadros de demência. Portanto, podemos considerar que as pessoas com QSM não apresentam prejuízos e/ou limitações na realização de suas tarefas cotidianas ${ }^{19}$, podendo relacionar, de modo satisfatório, com a amostra deste estudo.

As QSM são conhecidas por fazerem parte do cotidiano de idosos, sendo expressas por estes com base na autopercepção do próprio desempenho cognitivo ${ }^{3}$. De acordo com os resultados observados na Tabela 2, a amostra deste estudo apresentou um elevado número de queixas de memória, mensuradas por meio do Questionário de Memória Prospectiva e Retrospectiva (QMPR). Nota-se, então, um considerável número de queixas totais, tendo destaque as queixas de memória prospectiva (voltadas para a realização de intenções futuras). Este dado confirma uma das hipóteses aqui levantadas cujas queixas de memória prospectiva tendem a aparecer com maior frequência se comparadas às retrospectivas.

A respeito da diminuição da memória episódica no decorrer do processo de envelhecimento, estudos indicam que esse declínio pode ser explicado em partes pelo uso ineficiente de estratégias de memória ${ }^{20}$. Dixon e Hultsch $^{21}$ apontam que essas estratégias dividem-se em duas categorias, a saber, internas e externas. As estratégias de memória internas estão relacionadas com o ato de codificar ou recuperar mentalmente determinados fatos (ensaios, imagens), e, as externas, voltadas para os auxiliares de funcionamento de memória (uso de calendários, blocos de notas, lembretes, dentre outros). 
Blumenfeld e Ranganath ${ }^{22}$ sugerem que a utilização de estratégias exige o auxílio de processos de controle que regularizam as funções mnemônicas por meio da seleção, monitoramento e organização de informações durante a codificação. Estudos evidenciaram que o uso de estratégias internas reduz com o avançar da idade (no cotidiano e nas tarefas de codificação), já Touron et al ${ }^{23}$ não identificaram os efeitos da idade sobre tais estratégias, acreditando que um maior nível de reserva cognitiva possibilite o uso mais eficaz de estratégias de memória.

$\mathrm{Na}$ Tabela 3 destaca-se a significativa correlação entre as queixas de memória totais, prospectivas e retrospectivas, com o uso de estratégias. Esta afirmação vem confirmar outra hipótese do estudo de que as QSM estão significativamente correlacionadas com o uso de estratégias. Estudos como o de Nikita et al. ${ }^{24}$, em que se investigou o efeito do treinamento de estratégia de memória em idosos, aponta a importância de intervenções direcionadas ao uso de estratégias cognitivas com considerável redução das QSM na vida de pessoas idosas. Tais pesquisas confirmam a relevância do presente estudo ao buscar conhecer a relação entre as QSM e o uso de estratégias cognitivas, ampliando as pesquisas científicas sobre a temática, além da sua aplicabilidade prática e clínica.

Na presente pesquisa, também foi realizada uma divisão de grupos entre aqueles que apresentaram elevado e reduzido uso de estratégias de memória para um teste comparativo de variáveis correlatas, conforme apresentado anteriormente na Tabela 4. Observou-se que aqueles que demonstraram o alto uso de estratégias cognitivas, apresentaram um maior índice de queixas, tanto totais como prospectivas e retrospectivas. Com isso, pode-se concluir que quanto maior forem as queixas de memória relatadas, maior será o uso de medidas compensatórias; e quanto maior for o uso dessas medidas, melhor será a percepção do idoso acerca das queixas. Este achado correlaciona-se com outro estudo de Nikita et al. ${ }^{25}$, em que idosos com QSM implementaram maior uso de estratégias na vida diária.

O presente estudo apresentou limitações como o número reduzido de sujeitos da amostra. Sugere-se que futuras pesquisas invistam em intervenções com treinamentos para o uso de estratégias cognitivas em idosos saudáveis, que apresentem relatos de queixas subjetivas de memória, para melhor investigação dos efeitos e impactos das medidas compensatórias; bem como enfatizem as queixas de memória prospectivas, já que estas são tidas como corriqueiras na vida cotidiana do público em foco; além da realização de estudos longitudinais, para uma melhor compreensão das manifestações, ocorrências e progressos das QSM na vida das pessoas idosas.

\section{CONSIDERAÇÕES FINAIS}

O presente estudo teve por objetivo verificar a correlação entre as Queixas Subjetivas de Memória (QSM) evidenciadas pelo Questionário de Memória Prospectiva e Retrospectiva (QMPR) e as variáveis: Escolaridade (E), Reserva Cognitiva (RC), Funcionalidade (FAQ) e o uso de Estratégias (MMQ). Para tal alcance foi necessário realizar avaliações cognitivas dos sujeitos participantes da amostra.

Em síntese, percebeu-se correlação significativa entre as variáveis: Escolaridade (E), Reserva Cognitiva (RC), Memória Prospetiva e Retrospectiva (QMPR) e Funcionalidade (FAQ) com o uso de estratégias (MMQ). Destacou-se a alta prevalência de QSM nos idosos do estudo, tendo ênfase as queixas de memória prospectivas em detrimento das retrospectivas, o que veio a corroborar com a literatura referenciada. Compreendeu-se também que os idosos que fazem o alto uso de estratégias na vida diária apresentam uma melhor percepção sobre as queixas relatadas no cotidiano. A amostra da pesquisa é diversa, abrangendo uma multiplicidade de idosos com diferentes níveis de escolaridade, condições socioeconômicas e boa funcionalidade, o que a torna bastante significativa, mesmo sendo considerado como fator limitante o número de participantes.

A relevância dos resultados consiste nas contribuições para uma melhor compreensão sobre as queixas subjetivas de memória e o uso de estratégias na vida das pessoas idosas, favorecendo um entendimento ampliado, além de maiores subsídios para o campo da ciência. Sugerem-se pesquisas longitudinais e a realização de maiores estudos de base interventiva, que proporcione o treino de estratégias cognitivas em idosos que apresentem relatos de QSM, contribuindo para um melhor bem-estar e qualidade de vida.

Contribuições dos autores: Rafael Gomes Firmino - Elaboração do manuscrito, coleta de dados, análise e interpretação. Isabela Lemos Arteiro Ribeiro Lins - Participação na elaboração do projeto de pesquisa, supervisão e revisão crítica do manuscrito. Bernardino Fernandes Calvo - Participação na elaboração do projeto de pesquisa, supervisão e análise dos dados estatísticos. 


\section{REFERÊNCIAS}

1. IBGE. Instituto Brasileiro de Geografia e Estatística. Pesquisa nacional por amostra de domicílios contínua. Disponível em: https://biblioteca.ibge.gov.br/visualizacao/livros/ liv101566 informativo.pdf.

2. Howieson DB, Mattek N, Dodge HH, Erten-Lyons D, Zitzelberger T, Kaye JA. Memory complaints in older adults: Prognostic value and stability in reporting over time. SAGE Open Med.2015;3: Article 05031211557479, 2015. doi:10.1177/2050312115574796.

3. Montejo P, Montenegro M, Fernández MA, Maestú F. Subjective memory complaints in the elderly: Prevalence and influence of temporal orientation, depression and quality of life in a population-based study in the city of Madrid. Aging Mental Health. 2011;15(1) 85-96.

4. Balash Y, Mordechovich M, Shabtai H, Giladi N, Gurevich T, Korczyn AD. Subjective memory complaints in elders: depression, anxiety, or cognitive decline? Acta Neurol Scand. 2013;127:344-50. doi: 10.1111/ane.12038.

5. Perquin M, Diederich N, Pastore J, Lair M-L, Stranges S, Vaillant $\mathrm{M}$. Prevalence of dementia and cognitive complaints in the context of high cognitive reserve: a population-based study. PloS One. 2015;10(9).e0138818.

6. Jessen F, Wolfsgruber S, Weese B, Bickel H, Mösch E, Kaduszkiewicz $\mathrm{H}$, et al. AD dementia risk in late MCI, in early MCI, and in subjective memory impairment. Alzheimers Dement. 2014;10(1):76-83. doi: 10.1016/j.jalz.2012.09.017.

7. West RL, Thorn RM. Goal-setting, self-efficacy, and memory performance in older and younger adults. Exp Aging Res. 2001;27(1):41-65. doi: 10.1080/03610730126109.

8. Salthouse TA. Theoretical perspectives on cognitive aging. Hillsdale, NJ: Erlbaum; 1991.

9. Assis GM, Barreto KML, Assis LO. Terapia ocupacional em gerontologia. In: Freitas EV, et al., organizadores. Tratado de geriatria e gerontologia. 4a ed. Rio de Janeiro: Guanabara Kooga; 2017. p.3138-50.

10. Brucki, SMD, Nitrini R, Caramelli P, Bertolucci PHF, Okamoto $\mathrm{IH}$. Sugestões para o uso do miniexame do estado mental no Brasil. Arq Neuro-Psiquiatr. 2003;61(3-B):777-81. https://doi. org/10.1590/S0004-282X2003000500014.

11. Smith G, Della Sala S, Logie, RH, Maylor EA. Prospective and retrospective memory in normal ageing and dementia: a questionnaire study. Memory. 2000;8(5):311-21. DOI: $10.1080 / 09658210050117735$.

12. Folstein MF, Folstein SE, Mc Hugh PR. Mini-mental state: a practical method for grading the cognitive state of patients for the clinicians. J Psychiatr Res. 1975;12(3):189-98. doi: 10.1016/0022-3956(75)90026-6.

13. Pfeffer RI, Kurosaki TT, Harrah Jr CH, Chance JM, Filos $\mathrm{S}$. Measurement of functional activities in older adults in the community. J Gerontol. 1982;37:323-329. doi: 10.1093/ geronj/37.3.323.

Recebido em: 30.04.2019

Aceito em: 17.01.2020
14. Zigmond AS, Snaith RP. The hospital anxiety and depression scale. Acta Psychiatr Scand. 1983;67(6):371-70. doi: 10.1037/ t03589-000.

15. Rami L, Valls-Pedret C, Bartrés-Faz D, Caprelle C, Solé-Padulles $\mathrm{C}$, Castelvi M, et al. Cuestionario de reserva cognitiva. Valores obtenidos en población sana y con enfermedad de Alzheimer. Rev Neurol. 2011;52:195-201. doi: https://doi.org/10.33588/ rn.5204.2010478.

16. Simon et al. Metamemória e envelhecimento: propriedades psicométricas da versão brasileira do questionário multifatorial de memória para idosos. Dement Neuropsychol. 2016;10(2):113-26. https://doi.org/10.1590/S1980-5764-2016DN1002007.

17. Ginó S, Mendes T, Maroco J, Ribeiro F, Schmand B, Mendonça A, Guerreiro M. Memory complaints are frequent but qualitatively different in young and elderly healthy people. Gerontology. 2010;56(3):272-7. doi: 10.1159/000240048.

18. Organização Mundial da Saúde (OMS). Como usar a CIF: Um manual prático para o uso da Classificação Internacional de Funcionalidade, Incapacidade Física e Saúde (CIF). Versão preliminar para discussão. Out. Genebra: OMS; 2013.

19. Bajo R, Castellanos NP, Lópes ME, Ruiz JM, Montejo P, Montenegro M, et al. Early disfunction of functional connectivity in healthy elderly with subjective memory complaints. AGE. 2012;34:497-506. doi: 10.1007/s11357-011-9241-5.

20. Shing YL, Werkle-Bergner M, Brehmer Y, Müller V, Li S-C, Linddenberger U. Episodic memory across the lifespan: the contributions of associative and strategic components. Neurosci Biobehav Rev. 2010;34(7):1080-91. https:// doi.org/10.1016/j.neubiorev.2009.11.002.

21. Dixon RA, Hultsch, DF. Metamemory and memory for text relationships in adulthood: A cross-validation study. J Gerontol. 1983;38(6):689-94. https://doi.org/10.1093/geronj/38.6.689.

22. Blumenfeld RS, Ranganath C. Prefrontal cortex and longterm memory encoding: an integrative review of findings from neuropsychology and neuroimaging. Neuroscientist. 2007;13(3):280-91. doi: 10.1177/1073858407299290.

23. Touron DR, Oransky N, Meier ME, Hines JC. Metacognitive monitoring and strategic behaviour in working memory performance. Q J Exp Psychol (Hove). 2010;63(8):1533-51. doi: 10.1080/17470210903418937.

24. Nikita LF, Overdoro EJ, Fasottil L, Claassen JAHR, Kessels RPC, Oosterman JM. Memory strategy training in older adults with subjective memory complaints: a randomized controlled trial. J Int Neuropsychol Soc. 2018;24(10):1110-20. doi: 10.1017/ S1355617718000619.

25. Nikita LF, Overdoro EJ, Fasottil L, Claassen JAHR, Kessels RPC, Oosterman JM. Memory strategy use in older adults with subjective memory complaints. Aging Clin Exp Res. 2017;29:1061-5. doi: 10.1007/s40520-016-0635-1. 